\title{
Papel del capital intelectual en la calidad de las Instituciones de Educación Superior en Colombia
}

Nancy Arrieta-Reales

orcid.org/0000-0003-3342-0194

Universidad Simón Bolívar de

Barranquilla, Colombia

narrieta1@unisimonbolivar.edu.co
Gladys Gaviria-García

orcid.org/oooo-0o01-8377-7701

Universidad Simón Bolívar de

Barranquilla, Colombia

ggaviria@unisimonbolivar.edu.co

\section{José Consuegra-Machado}

orcid.org/00oo-0002-4479-6632

Universidad Simón Bolívar de

Barranquilla, Colombia

jrconsuegra@unisimonbolivar.edu.co

\section{Resumen}

Se presenta un análisis bibliográfico sobre el papel de la gestión del capital intelectual en la calidad de las Instituciones de Educación Superior colombianas (IES), realizado a partir de la revisión sistemática de artículos publicados en revistas indexadas en Scielo, Latindex, PubMed, Redalyc y Dialnet, entre 1996 a 2015 documentos de la UNESCO y libros editados en Argentina en 1997, Valencia España en 2009 y 2011, Bogotá en 1993 y en Estados Unidos en 1999. Fueron seleccionadas 45 referencias bibliográficas. El análisis histórico mostró que los modelos de capital intelectual existentes responden a las necesidades de las empresas de consumo y no a las de las IES. Otro resultado tiene que ver con los componentes que integran la calidad de la educación superior y cómo un gran número de ellos hacen referencia a la gestión del capital intelectual, entre los cuales se encuentran el fortalecimiento de procesos de investigación e innovación en las IES. Por lo tanto, se hace necesario contar con un nuevo sistema de gestión del capital intelectual que pudiese responder a las funciones sustantivas de las IES con suficiente amplitud como lo exige la naturaleza del manejo del conocimiento y de esa manera fortalecer sus procesos de calidad para un mayor reconocimiento y posicionamiento en la sociedad.

\section{Palabras clave}

Educación universitaria; control de calidad; gestión del conocimiento; calidad de la educación; Colombia (Fuente: Tesauro de la Unesco). 


\title{
The Role of Intellectual Capital in the Quality of Colleges and Universities in Colombia
}

\begin{abstract}
A bibliographic analysis is presented on the role management of intellectual capital plays in the quality of Colombian institutions of higher education (IHE). It is based on a systematic review of articles that were published in journals indexed in Scielo, Latindex, PubMed, Redalyc and Dialnet, between 1996 to 2015, UNESCO documents and books published in Argentina in 1997, in Valencia, Spain in 2009 and 2011, in Bogotá in 1993, and in the United States in 1999. Forty-five (45) bibliographical references were selected. The historical analysis shows existing intellectual-capitalmanagement models respond to the needs of consumer companies, but not to those of IHES. Another result concerns the components that make up the quality of higher education and how many of them refer to intellectual capital management, including a build-up in research and innovation processes at IHEs. Therefore, a new system for managing intellectual capital is needed, particularly one that responds to the fundamental functions of IHEs and has enough scope, as required by the nature of knowledge management, to be able to strengthen their quality processes with an eye towards greater recognition and positioning in society.
\end{abstract}

\section{Keywords}

University education; quality control; knowledge management; quality of education; Colombia (Source: Unesco Thesaurus). 


\section{Papel do capital intelectual na qualidade das Instituições de Ensino Superior na Colômbia}

\section{Resumo}

Apresenta-se uma análise bibliográfica sobre o papel da gestão do capital intelectual na qualidade das Instituições de Ensino Superior colombianas (IES), realizado a partir da revisão sistemática de artigos publicados em revistas indexadas em SciELO, Latindex, PubMed, Redalyc e Dialnet, entre 1996 e 2015, documentos da Unesco e livros editados na Argentina em 1997, em Valencia (Espanha) em 2009 e 2011, em Bogotá em 1993 e, nos Estados Unidos, em 1999. Foram selecionadas 45 referências bibliográficas. A análise histórica mostrou que os modelos de capital intelectual existentes respondem às necessidades das empresas de consumo e não às IES. Outro resultado se refere aos componentes que integram a qualidade do ensino superior, e como um grande número deles fazem referência à gestão do capital intelectual, entre os quais se encontram ofortalecimento de processos de pesquisa e inovação nas IES. Portanto, faz-se necessário contar com um novo sistema de gestão do capital intelectual que possa responder às funções substanciais das IES com suficiente amplidão como exige a natureza da gestão do conhecimento e, dessa maneira, fortalecer seus processos de qualidade para um maior reconhecimento e posicionamento na sociedade.

\section{Palavras-chave}

Colômbia; controle de qualidade; educação universitária; gestão do conhecimento; qualidade da educação (Fonte: Tesauro da Unesco). 
El presente artículo de revisión da a conocer cómo el capital intelectual se ha constituido en el intangible que genera valor en las empresas y tiene incidencia en la calidad de las instituciones de educación superior (IES). Ello ha conllevado la creciente necesidad de establecer procesos que garanticen su adecuada gestión, orientados a incrementar su producción, protección y distribución. Uno de los aspectos que han provocado una nueva forma de mirar al capital intelectual es la revolución tecnológica y la globalización de los mercados, que han impactado en todos los ámbitos de la sociedad, el gobierno y la economía en general, en los cuales el conocimiento debidamente agenciado se constituye en un elemento clave del desarrollo económico y de las instituciones, lo que hace necesario medirlo dentro de las empresas.

La educación superior, considerada como empresa social, pletórica de intangibles, no es ajena a los cambios producidos por la globalización ni al valor que el conocimiento ha adquirido para el mejoramiento y el crecimiento de las sociedades. De hecho, su esencia es la de motivar la producción de conocimiento y transferirlo, para lograr la transformación de la sociedad y el mejoramiento de las ciencias. Igualmente, incide el impacto de la masificación de las tecnologías de la información y la comunicación, que han llevado a su transformación, lo cual permite el posicionamiento de los países y regiones en el contexto geopolítico.

Sin embargo, los modelos de gestión de capital intelectual han sido creados para las empresas del sector productivo y no para la educación superior. Las IES adaptan sus necesidades a los modelos existentes, pero no son completamente efectivas, ya que los fines son distintos. Para el sector productivo, estos son netamente económicos: producir más y mejor para ganar más dinero, más clientes, más reconocimiento, mientras que para el sector educación el fin es netamente social. Su misión es formar talento humano que responda efectivamente a las necesidades del contexto, con idoneidad. De igual manera, se quiere obtener un mayor posicionamiento y reconocimiento de su calidad en el ámbito social, tanto local como nacional e internacional, por ello la adecuada gestión del capital intelectual es un pilar fundamental de ese logro. La calidad de la educación superior se ha constituido en un reto que las IES del mundo y Colombia han asumido como manera de garantizar la formación de un talento humano acorde con lo que la sociedad necesita, capaz de mejorar las situaciones adversas y transformarlas.

\section{Materiales y métodos}

Estudio con enfoque sistémico, fundamentado en el rol que cumple el capital intelectual en la calidad de las instituciones de educación superior. Se hizo una búsqueda en bases bibliográficas y bibliotecas especializadas, en Scielo, Elsevier, Pubmed, Latindex, Redalyc, Dialnet, Unesco, Repositorio del Ministerio de Educación Nacional de Colombia. También fueron consultados libros referentes al tema. Se tuvieron en cuenta los criterios de calidad y pertinencia de las referencias revisadas (tabla 1).

Tabla 1. Criterios de calidad y pertinencia de los documentos

\begin{tabular}{|c|}
\hline Calidad \\
\hline Clasificación del artículo \\
\hline Fecha de publicación \\
\hline Número de citación de los artículos \\
\hline Índice de impacto de la revista \\
\hline Pertinencia \\
\hline Concordancia con las palabras clave \\
\hline Contenido de los documentos \\
\hline Tendencias actuales de la gestión en educación \\
superior \\
\hline
\end{tabular}

Fuente: Elaboración de los autores. 
Para la revisión se tuvieron en cuenta las palabras clave que orientaban la temática de referencia. La pesquisa se realizó en cuatro fases, que integraron: planeación, búsqueda, organización y selección y redacción del artículo (tabla 2).

Inicialmente fueron revisadas 100 referencias, luego se preseleccionaron 55 documentos y finalmente se eligieron un total de 45 referencias para la construcción final del artículo (tabla 3).

\section{Conocimiento y capital intelectual: breve reseña histórica}

El conocimiento ha sido de mucha importancia en todos los tiempos como una tarea incesante del hombre por sobrevivir, al tener la necesidad de ir encontrando las respuestas ante los cambios que el mundo le ha ido imponiendo, lo que lo ha llevado a evolucionar y trascender en la historia. Aunado a ello, el lenguaje ha jugado un papel preponderante,

Tabla 2. Fases de elaboración de la revisión bibliográfica

\begin{tabular}{|c|c|c|c|c|}
\hline No. & Fases & Procedimiento & Resultados & Medio \\
\hline 1 & $\begin{array}{l}\text { Planeación } \\
\text { de la revisión } \\
\text { bibliográfica. }\end{array}$ & $\begin{array}{l}\text { Organización de las fases de } \\
\text { revisión según criterios de los } \\
\text { investigadores. }\end{array}$ & $\begin{array}{l}\text { Establecimiento del cronograma con } \\
\text { las fases y fechas de las referencias. }\end{array}$ & Ficha bibliográfica \\
\hline 2 & $\begin{array}{l}\text { Búsqueda } \\
\text { bibliográfica. }\end{array}$ & $\begin{array}{l}\text { Consulta de bases de datos y } \\
\text { fuentes documentales. }\end{array}$ & $\begin{array}{l}\text { Planificación de estrategias para las } \\
\text { pesquisas. } \\
\text { Establecimiento de criterios de } \\
\text { elección para las búsquedas } \\
\text { bibliográficas. }\end{array}$ & $\begin{array}{l}\text { Selección de bases } \\
\text { de datos. }\end{array}$ \\
\hline 3 & $\begin{array}{l}\text { Organización y } \\
\text { selección de la } \\
\text { información. }\end{array}$ & $\begin{array}{c}\text { Elaboración de resúmenes de las } \\
\text { pesquisas. }\end{array}$ & Diseño el estado del arte. & $\begin{array}{l}\text { Preparativo del } \\
\text { documento. }\end{array}$ \\
\hline 4 & $\begin{array}{l}\text { Redacción del } \\
\text { artículo. }\end{array}$ & $\begin{array}{l}\text { Revisión de normas de } \\
\text { publicación. }\end{array}$ & $\begin{array}{l}\text { Selección de revistas para publicar el } \\
\text { producto elaborado. }\end{array}$ & $\begin{array}{l}\text { Diseño final del } \\
\text { paper. }\end{array}$ \\
\hline
\end{tabular}

Fuente: Elaboración de los autores.

Tabla 3. Revisión sistémica

\begin{tabular}{|c|c|c|c|c|}
\hline Tipo de referencias & $\begin{array}{c}\text { Referencias } \\
\text { revisadas }\end{array}$ & Bases de datos & Preseleccionadas & Seleccionadas \\
\hline \multirow{5}{*}{ Artículos } & \multirow{5}{*}{71} & Scielo & \multirow{5}{*}{35} & \multirow{5}{*}{32} \\
\hline & & Latindex & & \\
\hline & & PubMed & & \\
\hline & & Redalyc & & \\
\hline & & Dialnet & & \\
\hline \multirow{2}{*}{ Libros } & \multirow{2}{*}{19} & Elsevier & \multirow{2}{*}{13} & \multirow{2}{*}{7} \\
\hline & & Bibliotecas & & \\
\hline Normas & 4 & $\begin{array}{l}\text { Repositorio del } \\
\text { Ministerio de Educación } \\
\text { Nacional de Colombia }\end{array}$ & 2 & 2 \\
\hline Tesis & 4 & Redalyc & 3 & 2 \\
\hline Documentos & 2 & Unesco & 2 & 2 \\
\hline Total de referentes bibliográficos revisados & 100 & & 55 & 45 \\
\hline
\end{tabular}

Fuente: Elaboración de los autores. 
al ser la herramienta que le permitió comprender, interpretar y transmitir cada hecho o suceso que ha acontecido en el contexto sociocultural en el cual se encontraba inmerso.

De acuerdo con Candel y Walle (2007, 2001, citados en Perdomo, 2010), el ciclo dinámico del conocimiento se inició formalmente con Aristóteles, el cual estructuró y fundamentó las nociones del conocimiento práctico, teórico y productivo, que siglos más tardes fueron legitimadas sistemáticamente por Descartes, quien consideró que lo más importante es la duda metódica, ya que se debe dudar de todo lo que se ve y existe, del conocimiento, de lo que captan los sentidos e incluso del propio cuerpo. Esto lo concretó Kant al formular su teoría del conocimiento, en la que manifiesta que este surge del trabajo conjunto del pensamiento lógico propio del racionalismo con la experiencia sensorial avalada por el empirismo.

Como Kant, asimismo Hume, Hegel y Marx (Rosales, 2011; Ramírez, 2009) realizaron aportes encaminados a profundizar en los aspectos que integran el conocimiento. Kant indicaba que la razón humana tiene mucha importancia y consideraba que el conocimiento es limitado, de modo que su teoría no admite como realidad al objeto en sí, sino al propio sujeto, que es el portador de las formas y esquemas universales obtenidos por la experiencia. Hume coincidía con Kant en que el conocimiento se inicia con la experiencia, pero, en contraposición a él, veía a la mente como un agente pasivo, que solo se limita a recibir información sobre el objeto que observa. Hegel y Marx, ya en el siglo XIX, condujeron una nueva forma de conocimiento denominada materialismo dialéctico. Para esta corriente, solo eran posibles la conciencia, el espíritu y el conocimiento en cuanto el mundo es materia. Consideraban que lo que es racional es real y a la inversa. La materia es el sustrato de toda realidad y de esa manera privilegia e independiza lo material de lo subjetivo.

Años más tarde emergió una nueva corriente denominada pragmatismo, representada por Sanders
Peirce, el cual postulaba que solo es verdadero aquello que funciona, indicando con ello que las ideas sin acciones no son nada (en Perdomo, 2010). Otra corriente es la denominada fenomenología, cuyas bases fueron sentadas por Edmund Husserl, según Nonaka y Takeuchi (1999). En ella se da importancia a la experiencia consciente y directa, fundamentada en la investigación filosófica de la conciencia humana, en la relación entre el yo pensante y el mundo. Considera la fenomenología que la "conciencia pura" solo puede alcanzarse a través de la "reducción fenomenológica", lo cual permite analizar la intuición pura de su esencia. Seguidamente, en 1946 surge la corriente llamada existencialismo, uno de cuyos exponentes fue Jean-Paul Sartre, quien manifestó que "el acto humano debe estar definido por una intención" y que esta elección del fin "revela el mundo" (en Ehrenzweig, s.f.). Más adelante, hacia 1907, surge el pragmatismo. Uno de sus exponentes fue John Dewey, quien sostuvo que "las ideas no sirven de nada, excepto que se vuelvan acciones que se reacomodan y reconstruyen de alguna manera, sea en grande o en pequeña medida, el mundo en el que vivimos" (1975). Aquí la experiencia juega un papel preponderante para la obtención del conocimiento.

Lo anterior demuestra que encontrar una definición de conocimiento, ver cómo se construye y cómo este contribuye al mundo es algo que ha motivado a los estudiosos de todos los tiempos, y que, a pesar de ello, aún en la actualidad no hay una definición única. Existen coincidencias en que el conocimiento se construye y es en la mente y en el pensamiento donde se logra estructurar la información que es tomada de la realidad emanada del contexto sociocultural y se la procesa, para luego ser transmitida a otros y de esa manera lograr impactar en el mundo.

Pero fue en la década de 1990 cuando, de acuerdo con Muzard (2011), la gestión del conocimiento se hizo más notoria, hecho que coincidió con la aparición de la obra de Peter Senge sobre la organización que aprende. Para él, una organización inteligente 
es igual a una organización que entiende, al hacer del aprendizaje una práctica continua.

La expresión capital intelectual con frecuencia se usa como sinónimo de intangibles, activos intangibles, recursos intangibles, propiedad intelectual, valores inmateriales, conocimiento, entre otros (Cano, Sánchez, González y Pérez, 2014). Su denominación de capital es debido a sus raíces económicas. Para Stewart (1997), por su parte, el capital intelectual es aquello que no se puede tocar pero que hace ganar dinero a las empresas, siendo esta una postura puramente mercantilista. Una definición más visionaria es la presentada por Drucker (1993), el cual resalta la importancia de la llegada de una nueva sociedad en la que dominan los activos del conocimiento y se compite por la distribución del Cl como una forma de lograr un mayor posicionamiento de las empresas en el mercado.

Años más tarde, Bontis (1996, citado por Naranjo y Chu, 2014, p. 32) presenta lo que desde su perspectiva es la estructura del capital intelectual y realiza un análisis referente a las relaciones de causa-efecto entre los elementos que la integran: capital humano, capital estructural y capital relacional, y su efecto en los resultados empresariales. Mientras que para Brooking (1997, p. 25), el Cl se divide en cuatro categorías: activos de mercado, activos de propiedad intelectual, activos centrados en el individuo y activos de infraestructura, Kaplan y Norton (2001) presentan una mirada más humanizada e integral del $\mathrm{Cl}$ e incluyen en su análisis los indicadores de gestión en el modelo Balanced Business Scorecard (Cuadro de Mando Integral - CMI), el cual integra cuatro perspectivas: a) financiera, referida a los indicadores financieros, flujos de caja, análisis de rentabilidad de cliente y producto, gestión de riesgo; b) del cliente, que identifica los valores relacionados con los clientes; c) de procesos internos, es decir, de satisfacción de clientes internos y clientes externos, por medio tanto de innovaciones y operaciones como de servicio posventa; d) del aprendizaje, referida a innovación, crecimiento, motivación y capaci- dad de aprender y crecer con base en la gestión de los empleados.

Una definición más integral, que trasciende incluso a la comunidad académica científica, ha sido la presentada por el profesor Bueno (2001), quien la define como "el conjunto de capacidades, destrezas y conocimientos de las personas que generan valor [...] para las comunidades científicas, las universidades, las organizaciones y la sociedad en general". Años más tarde, Bueno, Salmador y Merino presentaban una definición mucho más estructurada, según la cual:

El capital intelectual es la acumulación de conocimiento que crea valor o riqueza cognitiva poseída por una organización, compuesta por un conjunto de activos intangibles (intelectuales) o recursos y capacidades basados en conocimiento, que cuando se ponen en acción, según determinada estrategia, en combinación con el capital físico o tangible es capaz de crear valory competencias esenciales en el mercado. (2008)

Todo lo expresado lleva a afirmar la necesidad de consolidar organizaciones inteligentes, organizaciones que aprenden:

El precepto de una organización que aprende es hoy la panacea en términos de las nuevas olas de la gestión y la teoría organizacional, es por esto que el ente empresarial continuamente se adapta, tiende a reinventarse, se transforma y capitaliza efectivamente los flujos de conocimiento a través de prácticas de aprendizaje organizacional. (Rodríguez, Gutiérrez y Castillo, 2012)

Actualmente, Román (2005) y González y Rodríguez (2010) coinciden en que la generación de nuevo conocimiento en las empresas del siglo XXI ha promovido importantes cambios en la sociedad. Es por ello que adquiere gran importancia forjar capital intelectual, ya que permite la producción de conocimiento, al ser este el insumo primario de lo que se fabrica, se compra y se vende. Por ende, se hace nece- 
sario administrarlo correctamente, hallarlo, acrecentarlo, almacenarlo, venderlo y compartirlo. Se convierte entonces en una condición sine qua non para las empresas que deseen ser exitosas y competitivas, y ello las motiva a gestionar y desarrollar el control intelectual dentro de sus organizaciones.

Asimismo, Alarcón, Álvarez, Goyes y Pérez (2012) manifiestan que, en la gestión empresarial, la inexperiencia y el desconocimiento de los elementos intangibles la mayoría de veces son causa del desaprovechamiento de oportunidades de negocio cuya base son los recursos intangibles que pasan desapercibidos a sus directivos. Para Rodríguez (2006, p. 26), la relación económica de la producción ha cambiado del enfoque tradicional de tierra, capital y trabajo - activos tangibles que se deprecian en el tiempo- al derivado del procesamiento de la información y de la distribución y transferencia del conocimiento - activos intangibles que no se deprecian en el tiempo y, por el contrario, con su uso crecen-. De esa manera, las instituciones saludables generan y usan conocimiento, con lo cual hacen necesario realizar esfuerzos para efectuar una adecuada gestión del conocimiento.

Para que las empresas consigan mantenerse competitivas, de acuerdo con Cano et al. (2014), es importante lograr ubicar en el mercado servicios y productos innovadores. De ahí que la relación entre el capital intelectual y la capacidad de innovación cobren un papel determinante en la actual economía del conocimiento. En ese sentido, Dutta, Lanvin y Wunsch-Vincent (2014) indicaron que la mejora de las habilidades en las personas es una de las maneras más efectivas de elevar la innovación, la productividad y el crecimiento económico orientado a mejorar el bienestar social y al fomento de la igualdad, siendo la educación un factor fundamental para este logro.

De igual manera, el retener a los principales innovadores se constituye en un elemento esencial de competitividad para el desarrollo de los países. Es por ello que muchos gobiernos están poniendo la innovación en el centro de sus estrategias de crecimiento. De igual manera, Edvinsson (2013) y Dumay y Garanina (2013) consideraron que el capital intelectual podría ser denominado ciencia de sistemas, que estaría enfocada en el estudio, sistemático e interdisciplinario, orientado a identificar los recursos intelectuales para compartirlos, emplearlos y mantenerlos en los diferentes niveles, que van del individual al organizacional, social y global. Asimismo, para estos autores la investigación del capital intelectual deberá realizarse con un enfoque interdisciplinario, estrategia que permitirá ampliar los límites y hacer de esa manera que surjan nuevas formas de actuación en la práctica.

Por ende, Alarcón et al. (2012) han manifestado que los activos intangibles se constituyen en la mayor preocupación y ocupación de los directivos, al ser estos la fuente principal de la creación de valor en las organizaciones y ser la clave del éxito competitivo de las empresas. Este se convierte entonces en un campo de estudio cada vez más relevante, que involucra su identificación, determinación y evaluación desde diferentes puntos de vista, que integran la dirección empresarial y la responsabilidad social de la empresa. Asimismo, Cabaña y Garito (2003) manifiestan la necesidad de las empresas de identificar la naturaleza de los activos inmateriales o intangibles que integran el $\mathrm{Cl}$ y de orientados al establecimiento de medidas administrativas y de gestión eficaz que fomenten su crecimiento interno y externo, integrando medidas de control para garantizar su protección.

Es por ello que, de acuerdo con Hanisch, Linder, Mueller y Wald (2011, p. 148), se establece en la actualidad una economía caracterizada por el continuo cambio en la cadena de valor, en el que la fuerza de trabajo se ha movido a otros terrenos, como son el de servicios y el saber. 
Las IES no se encuentran ajenas a las situaciones planteadas, más aún siendo su esencia el fomento, distribución y manejo del conocimiento con un fin netamente social, que es contribuir al mejoramiento de la calidad de vida de los seres humanos y de la sociedad. Tal como lo han expresado Henao, López y Garcés (2014):

Que en la actual economía del conocimiento las IES compiten para liderar el desarrollo científico, técnico y tecnológico, mediante la formación integral de profesionales capacitados, y la promoción de la investigación y la innovación como actividades determinantes de su competitividad. En la búsqueda de mayores estándares de calidad, las IES se han volcado a la tarea de mejorar sus procesos, cualificar permanentemente su personal, innovar, investigar y producir conocimiento básico y aplicado que permita mejorar las condiciones de vida de toda la sociedad.

Reafirma lo anterior D. Bautzer (2010), al demostrar que las instituciones educativas no pueden ser administradas exclusivamente con modelos tradicionales de gestión empresarial, debido a su naturaleza y a la característica del servicio que ofrecen. Por tanto, sus directivas tienen el compromiso de de- sarrollar sistemas intuitivos y contingentes basados en el capital intelectual y, de esa manera, contar con herramientas que sustenten su crecimiento y posibiliten alternativas en situaciones de crisis, conservando su naturaleza. Núñez y Rodríguez coinciden con lo expresado anteriormente (2015, p. 68), al considerar que la gestión de recursos intangible y adecuada es el medio para alcanzar la misión, lo cual promueve el incremento de su capacidad de innovación y, por ende, la generación de mayor valor para la IES.

Sin embargo, los modelos existentes han sido creados para responder a las necesidades empresariales y no a las necesidades de la gestión del conocimiento y del capital intelectual de las instituciones de educación superior.

\section{La gestión del capital intelectual y la calidad de las instituciones de educación superior (IES)}

Lograr la calidad en los procesos educativos ha sido una gran preocupación de los gobiernos de todos los países. Es por ello que Brock (2007, citado por Freire y Teijeiro, 2010) consideró que la evaluación de la calidad tiene sus raíces en épocas pasadas, con características para cada período, descritas en la tabla 4, a continuación:

Tabla 4. Periodos de la evaluación de la calidad de la educación

\begin{tabular}{|c|c|c|}
\hline Periodo & \multicolumn{1}{c|}{ Características } \\
\hline Prerrenacentista & $\begin{array}{l}\text { La universidad tenía un carácter instrumental, al servicio de las élites urbanas (políticas, } \\
\text { religiosas e instrumentales), lo cual llevaba a que los contenidos fueran regulados, al igual que } \\
\text { los estándares de la práctica de la enseñanza, con el fin de alcanzar reconocimiento público. }\end{array}$ \\
\hline $\begin{array}{c}\text { Siglos XVII, XVIII e } \\
\text { inicios del XIX }\end{array}$ & $\begin{array}{l}\text { Con la corrupción en las universidades, los estándares de calidad eran bajos. El modelo de } \\
\text { universidad aplicado fue el francés, con mayor oportunidad de ingreso de personal y mayor } \\
\text { grado de cohesión política. Para lograr la titulación se debían cumplir los estándares mínimos, } \\
\text { las regulaciones y los estatutos de fundación; los programas eran diseñados para satisfacer las } \\
\text { necesidades de mano de obra de la burocracia. }\end{array}$ \\
\hline $\begin{array}{c}\text { Modelo universitario } \\
\text { británico de finales } \\
\text { del siglo XIX }\end{array}$ & $\begin{array}{l}\text { Hay una ligera presencia del Estado, en la que se da la política de concesión de becas y a las } \\
\text { nuevas instituciones de ciencia y tecnología se les brindan ayudas y financiación. }\end{array}$ \\
\hline Año 1919 & $\begin{array}{l}\text { Se crea la Comisión de Subvenciones Universitarias en el que se afianza el papel del Estado } \\
\text { en la universidad, lo que lleva a la consolidación de las instituciones de calidad en el siglo XXI. }\end{array}$ \\
\hline
\end{tabular}

Fuente: Elaboración de los autores a partir de Freire y Teijeiro (2010). 
Ya en el siglo XXI, en la Conferencia Mundial de Educación Superior de la Organización de las Naciones Unidas para la Educación, la Ciencia y la Cultura (Unesco), de 2009, llamada "Las nuevas dinámicas de la educación superior y de la investigación para el cambio social y el desarrollo", se dieron las indicaciones para que en la agenda educativa global se reflejara una serie de realidades que integran: la responsabilidad social de la educación, y el acceso, la calidad y la equidad, entre otros componentes. Los lineamientos apuntan a una educación superior contextualizada, globalizada, equitativa y de calidad donde la autonomía universitaria no exime a las IES públicas y privadas de que establezcan y se sometan a mecanismos regulatorios que garanticen y den fe de la calidad de los procesos formativos que allí se desarrollan. La calidad en la educación tiene diversas definiciones, para la Unesco el tema versa sobre:

La calidad abarca todas sus funciones y actividades principales: calidad de enseñanza, de formación e investigación, lo que significa calidad de su personal docente y de los programas, y calidad de aprendizaje como corolario de la enseñanza y la investigación. Aunque también implica prestarle atención a cuestiones que pertenecen a la calidad de los estudiantesy de la infraestructura, asi como del entorno académico. (2007)

Para el Consejo Nacional de Acreditación de Colombia, el concepto de calidad no difiere del presentado:

El concepto de calidad aplicado al bien público de la educación superior hace referencia a la sintesis de caracteristicas que permiten reconocer un programa académico específico o una institución de determinado tipo y hacer un juicio sobre la distancia relativa entre el modo como en esa institución o en ese programa académico se presta dicho servicio y el óptimo que corresponde a su naturaleza. (2011)

Todos esos aspectos relacionados con la calidad, unidos a un interés adecuado por un buen gobierno y administración, juegan un papel importante en la manera como funciona determinada institución, en la forma en que es evaluada y en la "imagen institucional" que puede proyectar hacia la comunidad académica y la sociedad en general. La evaluación comprende dos aspectos: la evaluación de los ajustes de los productos de cada proceso y la evaluación de los propios procesos (Tünnermann, 2006).

La educación superior, consciente del valor que el conocimiento ha adquirido para el mejoramiento y el crecimiento de las sociedades y de la importancia de una buena gestión del capital intelectual para su logro, tiene como eje fundamental — según Altbach, Reisberg y Rumbley (2009) - la mundialización, que lleva a la integración de la economía mundial, al fortalecimiento de las tecnologías de la información y comunicación (TIC) y a la conformación de redes de conocimiento, siendo la internacionalización la respuesta de las universidades a esa necesidad, las cuales según la Unesco (2009, p. 20), requieren suscitar el intercambio de conocimientos y opiniones cobijando la libertad académica bajo la protección de los derechos humanos,

Todo lo anterior — según Harman (2011) y Hazelkorn (2012), citados por Gacel-Ávila y Orellana (2013, p. 12) - ha estimulado a los gobiernos a la creación e implementación de políticas y estrategias de medición para la evaluación de la calidad de la educación superior. Entre los instrumentos se encuentran: la acreditación de programas e instituciones, los sistemas de aseguramiento de la calidad, los rankings nacionales y globales, entre otros. Sin embargo, Rojas (2012) ha manifestado que se ha hecho necesario pensar la manera como debe ser planeada y administrada la educación superior, para que se logre alcanzar los estándares internacionales, los cuales se han venido diseñando e implementando de acuerdo con su homólogo en el sector productivo, Modelo ISO. Es así como los gobiernos latinoamericanos vienen trabajando con ahínco en impulsar la calidad y procuran que esta sea pertinente al funcionamiento de los diferentes componentes del aparato social. 
En su Declaración, la Unesco (2009) recomienda a las instituciones de educación superior la formación de sus docentes para garantizar la calidad y propone que sean aplicados nuevos enfoques, tales como el uso de aprendizaje abierto y a distancia, las tecnologías de información y comunicación y de la investigación pedagógica, de modo que lleven al mejoramiento de las estrategias didácticas. También manifiesta que los criterios de calidad deben reflejar los objetivos globales de la educación superior, entre los cuales se encuentran: la meta de cultivar en los alumnos el pensamiento crítico e independiente y la capacidad de aprender a lo largo de toda la vida, criterios que estimulan la innovación y la diversidad. Las recomendaciones anteriores afianzan la necesidad de establecer modelos que lleven a la consolidación y manejo idóneo del capital intelectual en las IES y a que ello se convierta en una forma de articulación de la calidad académica, hacia el fortalecimiento del rol de la universidad en la solución de los problemas de la sociedad.

En Colombia, el Ministerio de Educación Nacional (2010) ha establecido el sistema de evaluación de la calidad teniendo en cuenta los lineamientos mundiales para tal fin, a través de una política que integra cuatro estrategias: 1) consolidación del Sistema de Aseguramiento de la Calidad en todos sus niveles; 2) implementación de programas para el fomento de competencias; 3) desarrollo profesional de los docentes y directivos; y 4) fomento de la investigación. Busca con ellas el fortalecimiento de las instituciones educativas, para que sean espacios donde todos puedan aprender, desarrollar competencias y convivir pacíficamente. Se observa que todos los lineamientos apuntan al fortalecimiento de la gestión del capital intelectual, al ser, según Nava y Mercado (2010), un pilar fundamental para los cambios tecnológicos, económicos y sociales, lo cual hace necesario reconocer, valorar, impulsar y gestionar este intangible, identificando sus elementos e indicadores. Son estos intangibles - capacidades y conocimientos - los que precisamente les otorgan a las empresas y especialmente a las IES ventajas competitivas y sostenibilidad en el tiempo.

\section{Conclusiones}

Los modelos que en la actualidad se presentan en la hoy llamada gestión del conocimiento nacieron con la llegada de un nuevo tipo de organización empresarial que se inserta en la nueva sociedad del conocimiento. Con objetivos, metas y valores diferentes a los del pasado paradigma, las organizaciones buscan adaptarse y sobrevivir en el actual orden mundial. Las Instituciones de educación superior (IES) no escapan a esta realidad, más aun cuando su fundamento, como es bien sabido, es la función social de la generación, trasmisión y aplicación del conocimiento de nuestras sociedades. Pareciera ser que desde hace mucho tiempo las universidades esperaban contar con un nuevo sistema de administración que verdaderamente pudiese responder a sus funciones sustantivas con suficiente amplitud, tal y como lo exige la naturaleza del manejo del conocimiento.

Por ende, se hace necesario replantear los manejos actuales de gestión del capital intelectual por las IES, las cuales se han acogido a los modelos estructurados para las empresas de consumo, por unos más agiles, eficaces e innovadores, cuyas características estén en correspondencia con las necesidades propias de la educación superior y lleven a la consolidación de la calidad de la IES y a un mayor reconocimiento y posicionamiento por la sociedad.

Se requiere que los directivos de las IES de hoy y del futuro se comprometan a desarrollar sistemas intuitivos y contingentes basados en el capital intelectual y que, de esa manera, cuenten con herramientas que sustenten su crecimiento y posibiliten alternativas en situaciones de crisis, conservando su naturaleza. Todo ello, unido a un apropiado interés por un buen gobierno y administración, orientado a motivar la producción de conocimiento, su transferencia al medio y su adecuada custodia, redunda en el fortalecimiento del rol que juega la universidad en la solución de los problemas de la sociedad. 
ISSN 0123-1294 | e-ISSN 2027-5358 | Educ.Educ. Vol. 20. No. 3 | Septiembre-diciembre de 2017 | pp. xx-xx.

Universidad de La Sabana | Facultad de Educación

\section{Referencias}

Alarcón, M., Álvarez, S., Goyes, J. y Pérez, O. (2012). Estudio y análisis del capital intelectual como herramienta de gestión para la toma de decisiones. Revista del Instituto Internacional de Costos, 10 (diciembre). Recuperado de http://www.revistaiic.org/articulos/num10/articulo3_esp.pdf

Altbach, Ph. G., Reisberg, L. y Rumbley, L. E. (2009). Tras la pista de una revolución académica: Informe sobre las tendencias actuales para la Conferencia Mundial sobre la Educación Superior organizada por la Unesco en 2009. Resumen ejecutivo. ED.2009/Conf.402/inf.6. París, 1-21. Recuperado de http://unesdoc.unesco. org/images/0018/001831/183168s.pdf

Álvarez, M., Chávez, M. y Moreno, S. (2009). El Balanced Scorecard, una herramienta para la planeación estratégica. Departamento de Contaduría y Finanzas, Instituto Tecnológico de Sonora. Recuperado de http:// www.itson.mx/publicaciones/pacioli/Documents/no66/17a-el_bsc_una_herramienta_para_la_planeacion_estrategicax.pdf

Arango, M., Pérez, G. y Gil, H. (2008). Propuesta de modelos de gestión de capital intelectual: una revisión. Contaduría Universidad de Antioquia, 52, 105-130.

Arias F., Castro, J. U. y Sánchez, J. B. (2010). Contribución del capital intelectual de la Universidad de Caldas a su capacidad investigativa. Tesis de grado, Maestría en Creatividad e Innovación en las Organizaciones, Universidad de Caldas. Recuperado de http://repositorio.autonoma.edu.co/jspui/bitstream/11182/261/1/ TESIS\%2OPDF.pdf

Augier, M. y Teece, D. J. (2007). dynamic capabilities and multinational enterprise: Penrosean insights and omissions. Management International Review, 47(2), 175-192. Recuperado de http://www.researchgate. net/publication/226085846_Teece_D.J._Dynamic_capabilities_and_multinational_enterprise_Penrosean_insights_and_omissions

Bautzer, D. (2010). La gestión del capital intelectual y su impacto en la educación superior. Innovación Educativa, 10(51) (abril-junio), 15-21. Recuperado de http://www.redaly c.org/articulo.oa?id=179421038002

Birchall, D. W. y Armstrong, M. (2004). Market understanding as a determinant of innovation success in SMEs. EBS Review (verano), 17-29.

Bontis, N. (1996). Modelo Universidad de West Ontario. Recuperado de http://bvs.sld.cu/revistas/aci/vol13_6_05/ acio60605.htm

Bravo, E. R. (2005). Identificación y caracterización de las capacidades dinámicas que intervienen en el contexto de la innovación de producto: (Estudio de Casos). Tesis doctoral, Universidad Politécnica de Cataluña, Barcelona. Recuperado de https://upcommons.upc.edu/pfc/bitstream/2099.1/6952/1/PT-Edna_Bravo.pdf

Bravo, E. D. (2009). Generación de las capacidades dinámicas mediante la innovación organizacional: un múl-

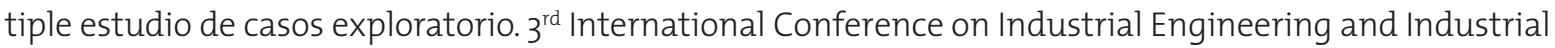


Management / XIII Congreso de Ingeniería de Organización Barcelona-Terrassa, Septiembre 2-4. Recuperado de https://upcommons.upc.edu/e-prints/bitstream/2117/7224/1/CIO-2009-07-05.pdf

Brooking, A. (1997). Capital intelectual, el principal activo de las empresas del tercer milenio (pp. 1-250). Buenos Aires: Paidós.

Bueno, C. E. (2001). Gestión del conocimiento en universidades y organismos públicos de investigación. Madrid: Dirección General de Investigación, Consejería de Educación, Comunidad de Madrid. Recuperado de http://www.madrimasd.org/informacionidi/biblioteca/publicacion/doc/16_GestionConocimientoUniversidadesOPIS.Pdf

Bueno, E., Salmador, M. P. y Merino, C. (2008). Génesis, concepto y desarrollo del capital intelectual en la economía del conocimiento: Una reflexión sobre el Modelo Intellectus y sus aplicaciones. Estudios de Economía Aplicada, 26(2) (agosto), 43-63.

Bueno, E., Del Real, H., Fernández, P., Lóngo, M., Merino, C., Murcia, C. y Salmador, M. (2011). Modelo Intellectus de medición, gestión e información del capital intelectual. Universidad Autónoma de Madrid. Recuperado de http://www.academia.edu/7807104/Modelo_Intellectus_Medici\%C3\%B3n_y_Gesti\%C3\%B3n_del_Capital_Intelectual

Bustos, E. (2011). La gestión del capital intelectual en las Instituciones de Educación Superior, ante el reto de la innovación en la sociedad del conocimiento. Instituto Politécnico Nacional, Escuela Superior de Comercio. Recuperado de http://www.colparmex.org/Revista/Art7/33.pdf

Cabañas, J. y Garito, M. A. (2003). Capital Intelectual, Gestión del Conocimiento y Comunicación: Factores Clave del Desarrollo de las Organizaciones en el siglo XXI. Universidad de Puebla Journal, 5 (9), 13-17.

Cano, M. C., Sánchez, G., González, M.y Pérez, J. C. (2014). El rol del capital intelectual en la innovación de las empresas. European Scientific Journal, 10(28) (octubre). Recuperado de http://eujournal.org/index.php/esj/ article/viewFile/4405/4202

Consejo Nacional de Acreditación (2011). Sistema Nacional de Acreditación en Colombia. Recuperado de http:// www.cna.gov.co/1741/article-186365.html

Dewey, J. (1975). Democracia y educación. Madrid: Morata.

Drucker, P. (1993). La gerencia en la sociedad futura. Bogotá: Norma.

Dumay, J. y Garanina, T. (2013). Intellectual capital research: a critical examination of the third stage. Journal of Intellectual Capital, 14(1), 10-25. Recuperado de http://www.emeraldinsight.com/doi/abs/10.1108/1469193 1311288995 ?journalCode $=$ jic

Dutta, S., Lanvin, B. y Wunsch-Vincent, S. (2014). The Global Innovation Index 2014: The human factor in innovation. Johnson Cornell University, Insead y World Intellectual Property Organization. Recuperado de https://www.globalinnovationindex.org/userfiles/file/reportpdf/Gll-2014-v5.pdf 
ISSN 0123-1294 | e-ISSN 2027-5358 | Educ.Educ. Vol. 20. No. 3 | Septiembre-diciembre de 2017 | pp. xx-xx.

Universidad de La Sabana | Facultad de Educación

Ehrenzweig, D. (s.f.). Libertad y acción responsable en Jean-Paul Sartre. Posibles factores reconfigurantes para superar el desencanto del hombre posmoderno. Recuperado de http://www.academia.edu/16107626/ LIBERTAD_Y_ACCI\%C3\%93N_RESPONSABLE_EN_JEAN-PAUL_SARTRE

Edvinsson, L. (2013). IC 21: reflections from 21 years of IC practice and theory. Journal of Intellectual Capital, 14(1), 163-172. Recuperado de http://www.emeraldinsight.com/doi/abs/10.1108/14691931311289075

Freire, M. J. y Teijeiro, M. (2010). Revisión histórica de garantía de la calidad externa en las instituciones de educación superior. Recuperado de http://www.scielo.org.mx/scielo.php?pid=\$0185-27602010000300007 \&script=sci_arttext

Gacel-Ávila, J. y Orellana, N. (2013). Educación Superior. Gestión, innovación e internacionalización. Valencia: JPM.

González, J. J. y Rodríguez, M. (2010). Modelos de Capital Intelectual y sus indicadores en la universidad pública. Cuadernos de Administración, 43, 113-128. Recuperado de http://www.scielo.org.co/pdf/cuadm/n43/ n43ag.pdf

Hanisch, B., Linder, F., Mueller, A. y Wald, A. (2011). Knowledge management in project environments. Journal of Knowledge Management, 13(4). Recuperado de http://arrow.dit.ie/cgi/viewcontent.cgi?article=1003\&con text=scschcomart

Henao García, E., López González, M. y Garcés Marín, R. (2014). Medición de octubre 21 de 2014. Capacidades en investigación e innovación en instituciones de Educación Superior: Una mirada desde el enfoque de las capacidades dinámicas. Entramado, 10(1) (enero-junio), 252-271. Recuperado de http://www.redaly c.org/ pdf/2654/265431574016.pdf

Kaplan, R.y Norton, D. (2001). Cómo utilizar el Cuadro de Mando Integral para implantary gestionar su estrategia (2 ed., pp. 100-348). Barcelona: Harvard Business School.

Ministerio de Educación Nacional (2010). Sistema de aseguramiento de la calidad de la educación superior. [Mineducación, portal web, 10 de junio.] Recuperado de http://www.mineducacion.gov.co/1621/w3-article-235585.html

Muzard, J. (2011). La evolución de la gestión del conocimiento en las organizaciones [Applied-intelligence-Atelier, Montreal, QC, Canadá.] Recuperado de http://www.a-i-a.com/auladigital/ArticuloGC-JM-ESP.pdf

Nava, R. M. y Mercado, P. (2010). Evaluación de la calidad métrica para indicadores de capital intelectual generados a partir de bases estadísticas. Revista de Educación Superior, 39(155). Recuperado de http://www. scielo.org.mx/scielo.php?pid=S0185-27602010000300006\&script=sci_arttext

Naranjo, C. y Chu, M. (2014). El capital intelectual en la Universidad Autónoma de Manizales. Tesis de Maestría en Administración de Negocios, Facultad de Estudios Sociales y Empresariales. Recuperado de http://repositorio.autonoma.edu.co/jspui/bitstream/11182/796/1/Documento\%2OFinal\%2O-\%2OM\%C3\%Bзnica\%2O Andrea\%2oChu\%2oSalgado.pdf 
Nonaka, I.y Takeuchi, H. (1999). La organización creadora de conocimiento. Cómo las compañías japonesas crean la dinámica de la innovación. Oxford University Press.

Núñez-Guerrero, Y. M., Rodríguez-Monroy, C. (2015). Gestión de recursos intangibles en instituciones de educación superior. RAE - Revista de Administração de Empresas, 55 (enero-febrero) Recuperado de http://www. redaly c.org/articulo.oa?id $=155133820006$

Perdomo Ch., G. (2010). La triada de la organización postmoderna: sujeto, poder, conocimiento. Recuperado de https://core.ac.uk/download/pdf/6552292.pdf

Ramírez, A. (2009). La teoría del conocimiento en investigación científica: una visión actual. Anuario de la Facultad de Medicina, 70(3), 217-24. Recuperado de http://www.scielo.org.pe/pdf/afm/v7on3/a11v7on3.pdf

Rodríguez, D. (2006). Modelos para la creación y gestión del conocimiento: una aproximación teórica. Educar,37, 25-39. Recuperado de http://ddd.uab.cat/pub/educar/O211819Xn37/O211819Xn37p25.pdf

Rodríguez, M. T., Gutiérrez, J. J. y Castillo, B. (2012). Estudio de aprendizaje organizacional de las microempresas de Sogamoso, Boyacá. Económicas CUC, 33(1), 81-102. Recuperado de http://revistascientificas.cuc.edu.co/ index.php/economicascuc/article/view/185

Rojas, J. M. (2012). Gestión educativa en la sociedad del conocimiento. Bogotá: Cooperativa Editorial Magisterio.

Román, N. (2005). Capital intelectual. Generador de éxito en las empresas. Visión Gerencial, 2(3), 67-79. Recuperado de http://www.saber.ula.ve/bitstream/123456789/25076/2/articulo6.pdf

Rosales, A. M. (2011). Los sistemas de Kant y Hegel. [Teológicamente, blog.] Recuperado de http://teologicamente.com/2011/05/los-sistemas-de-kant-y-hegel/

Tünnermann, C. (2006). Pertinencia y calidad de la educación superior, procesos de acreditación institucional y sistemas de acreditación (pp. 1-30). Universidad Rafael Landivar. Lección inaugural. Guatemala, 31 de enero. Recuperado de http://www.udea.edu.co/portal/page/portal/BibliotecaPortal//nformacionlnstitucional/Autoevaluacion/SistemaUniversitarioExtension/PertinenciaCalidadEducacionSuperior-CarlosTunnermann.pdf

Unesco (2007). Educación de calidad para todos: un asunto de derechos humanos. Recuperado de http://unesdoc.unesco.org/images/0015/001502/150272s.pdf

Unesco (2009). World Conference on Higher Education 2009 Final Report. París, 5 a 8 de julio, 1-156. Recuperado de http://www.unesco.org/education/WCHE2009/comunicado_es.pdf 